\title{
Glimpse of Contemporary Clinical Perspective on "Mindfulness Based Cognitive Behavioral Therapy" in Various Health Dimensions
}

\author{
Bemina JA ${ }^{1}$, Theraza Mathais ${ }^{2}$ \\ ${ }^{1} \mathrm{PhD}$ Scholar (Rajiv Gandhi University of Health Sciences), Laxmi Memorial College of Nursing, Balmatta, \\ Mangaluru, Karnataka, India. \\ ${ }^{2}$ HOD, Department of Mental health Nursing, Laxmi Memorial College of Nursing, Balmatta, Mangaluru , \\ Karnataka, India. \\ Corresponding Author: Bemina JA
}

DOI: https://doi.org/10.52403/ijrr.20220233

\begin{abstract}
Mindfulness-based cognitive therapy (MBCT) is a modified form of cognitive behavioral therapy that associates with mindfulness practices such as meditation and breathing exercises. Initially emerged as an intervention for relapse prevention in people with recurrent depression, it has since been connected to various psychiatric conditions and also has also shown marked effectiveness in various physical conditions. This paper aims to briefly describe the perspectives of MBCT and its presumed mechanism of action, and to review its effectiveness on different mental and physical wellbeing. The main therapeutic goal of MBCT is to interrupt automatic processes and teach the participants to focus less on reacting to incoming stimuli, and instead accepting and observing them without judgment due to which they can ended up more mindful of their contemplations and dispositions and not get caught within the circle of cynicism by utilizing mindfulness and CBT techniques. This new strategy enables to gain more control of thoughts and emotions. There are different confirmations on the viability of mindfulness based cognitive behavioural intervention in individual's mental and physical heath dimension.
\end{abstract}

Key Words: Mindfulness-based cognitive therapy, Mindfulness, Cognitive Behavioral Therapy

\section{INTRODUCTION}

Intellectual health is vital to anybody's universal fitness and well-being, and mental illnesses are not unusual and treatable. Hence, much of what we do physically impacts us mentally it's essential to take note of both bodily fitness and mental health, which can help achieve ordinary health and set on a path to recuperation. A healthy way of life can help to prevent various problems that arise from deviated bodily and mental fitness. Wellbeing is a high-quality outcome, which is significant for humans and for lots sectors of society because it tells us that human beings perceive that their lives are going well. Has it integrated intellectual fitness (mind) and physical fitness (body), ensuing in more holistic techniques to sickness prevention and fitness advertising. Being mindful is being aware about thoughts, emotions, and the way one feels, both physically and mentally. It means being privy to your mind and emotions without judgment. We need to bear in mind that there is no "right" or "wrong" way to suppose or feel at any moment. Mindfulness is the exercise of purposely focusing attention on the existing moment, accepting it without judgment. The cultivation of mindfulness has roots in Buddhism, but most religions encompass a few kinds of 
prayer or meditation methods that facilitate or shift your thoughts far away from your typical preoccupations, closer to an appreciation of the moment and a bigger attitude to existence. It improves one's wellbeing by increasing the capacity for mindfulness and helps many attitudes that contribute to a happy lifestyle. Among its theorized advantages are objectivity, affect tolerance, greater flexibility, equanimity, improved attention and mental readability, emotional intelligence and the capability to relate to others and one's self with kindness, recognition and compassion. Being mindful makes it less difficult to have fun with the pleasures in lifestyles as they arise, and involvement in activities, creating a greater ability to deal with detrimental events. Mindfulness is thought to promote good health, and many studies have linked mindfulness to a reduction in depression and anxiety. For example, many mental health professionals have incorporated activities that promote mindfulness into their therapy sessions, and these activities have been shown to help reduce symptoms of depression, reduce stress, and improve the ability to control emotions, regardless of specific problems being addressed. In particular, MBCT can be used as a primary treatment modality or in combination with other forms of therapy. People with certain medical conditions may also benefit from MBCT.

\section{ORIGIN}

Buddhist traditions date back over 2500 years, and mindfulness techniques are based on them. There has been a growing interest in using mindfulness in the setting of Western medical therapies in recent decades. Mindfulness" originates from the Pali time period sati and in its Sanskrit counterpart smṛti. Sati/ Smṛti firstly intended "to remember", "to recollect" , "to bear in mind". The arisement of sati calls to thought the healthful dhammas such as the four organizations of mindfulness, the 5 faculties, the 5 powers, the seven awakening-factors, the noble eight-factored path, and the attainment of insight The Palilanguage student Thomas William Rhys Davids (1843-1922) first translated sati in 1881 as English mindfulness in sammā-sati "Right Mindfulness; the active, watchful mind". Sati is literally 'memory' however is used with reference to the continuously repeated phrase 'mindful and thoughtful' (sato sampajâno); and potential that recreation of thinking and steady presence of mind which is one of the responsibilities most regularly inculcated on a true Buddhist." ${ }^{2}$ The phenomenological nature of mindfulness can be found in most religious traditions, for example, Tafakkur in Islam, Kabala in Judaism and the rosary in Christianity. ${ }^{3}$ Mindfulness-Based Stress Reduction (MBSR) program at the University of Massachusetts to treat the chronically unwell and sparked the software of mindfulness ideas and practices in Medicine. Its exercise does not mean following any precise philosophical or non secular culture "Mindfulness movement" The origins of mindfulness within Buddhist philosophy and practice are investigated as preliminary to the treatment of contemporary perspectives of mindfulnessbased notions in recent secular therapeutic perspectives. $^{4}$ The two main approaches seen in recent years times are Mindfulnessbased Stress Reduction (MBSR) and Mindfulness-based Cognitive Therapy (MBCT) both are taught over a number of sessions.

\section{WHAT IS MINDFULNESS BASED COGNITIVE BEHA VIOURAL THERAPY}

Mindfulness is a broad and multidimensional concept which is at once a theoretical construct, a meditative practice and a psychological process. ${ }^{5}$

Mindfulness is described as a non elaborative, non-judgmental, present centered awareness in which each thought, feeling, or sensation that arises in the attention field is acknowledged and accepted as it is. ${ }^{6}$ 
Definition of mindfulness involves three qualities and three activities. The accompanying qualities are nonjudgmentally, with acceptance, allowing; in the present moment, with beginner's mind; and effectively. The three activities include observing, noticing, bringing awareness; describing, labeling, and noting; and participating. They posit three components of mindfulness: intention, attention, and attitude (IAA). ${ }^{7}$

MBCT comprises eight sessions with 12 participants for two hours per week. Since MBCT adapts MBSR, the exercises included in the programme are the same as MBSR, except lovingkindness meditation. One of the unique techniques in MBCT is Three-Minute Breathing Space. This technique consists of three steps: (1) focus on the internal experiences of asking "What is my experience right now?" to notice the sensations currently arising without judging, (2) pay attention to the sensations of the breath process, (3) broaden awareness to the whole body without judging. Clients are required to practice this technique every day, particularly when they feel overwhelmed. ${ }^{8}$

\section{How Does MBCT Work?}

Mindfulness can assist you to interrupt the automatic connection of negative mood, negative questioning and bodily sensations such as fatigue and "sluggishness" that frequently link, trigger Or reactivate a downward mood spiral.

Mindfulness approves you to "shift gears" from a mode of mind dominated by means of vital and judgmental wondering (likely to provoke and speed up downward temper spirals) to any other mode of thinking in which you experience the world directly, non-conceptually and nonjudgmentally.

Mindfulness presents access to some other approach to dealing with tough emotions and moods. Through mindfulness, you can find out that hard and undesirable ideas and feelings can be held in awareness, and considered from an altogether one-of-a- kind standpoint - a perspective that brings with it a feel of warmth and compassion to the struggling you are experiencing. Mindfulness can help you analyze how to be existing and admire the easy pleasures of daily life, join with yourself, and the trip of being alive. ${ }^{9}$

\section{MINDFULNESS TECHNIQUES}

Mindfulness is a technique you can learn to be fully present and engaged in the moment without judging anything. It can help you manage your thoughts, feelings; mental health and physical wellbeing. ${ }^{10}$ There are various techniques such as

Three Minutes of Mindfulness - First minute: During the first minute, try to absolutely notice your ideas and feelings. One has to attempt to center of attention and observe their thoughts and emotions as they occur. - Second minute: During the second minute, recognition to consist of bodily sensations. Awareness of ideas and emotions has to be maintained. - Third minute: In the final minute, consist of the space round you. Awareness of ideas and feelings has to be maintained as properly as the bodily sensations. Notice the room or area around. As the 0.33 minute comes to a close, deliver your attention lower back to your breath and slowly open your eyes.

The Raisin - In this exercising raisin is held in the palm. It is looked, examined, felt, smelled, tasted, word each and every aspect of it. Then the journey is described. This exercise is about cultivating cognizance and beginning to study to focal point on the here-and-now. It is about Being in the current moment.

The Park Bench - This park bench ride is analogous to an exercise that can be achieved with thoughts, feelings, physical sensations, and observations of the house around. It has to described next. 


\section{Breathing Techniques \\ Breathe of Fire:}

Kapalbhati Pranayama: Kapalbhati is a very active, pressured exhalation with a passive inhalation. Try starting with 2-3 rounds of 30 exhalations. Then, progressively extend the exhalations, have to experience comfortable.

\section{Nostril Alternating Breath: Nadi} Shodhana Pranayama: Closing off right nostril with the thumb and inhale into the left nostril. Next, closing off left nostril with forefinger (or some other finger), open the right nostril by removing thumb and exhale through the right nostril. It has to be tried doing 5 "rounds" when begin. As one sense greater comfortable, it has to tried 10 or 20 rounds.

Three Part Breath: Dirga Pranayama: Dirga Pranayama is known as the three phase breath due to the fact you are actively respiration into three parts of your abdomen. The first role is the low belly (just beneath the stomach button), the second position is the low chest (belly and lower $1 / 2$ of the rib cage), and the 0.33 function is the top chest and low throat (just above the pinnacle of the sternum). The breath is continuous, inhaled and exhaled thru the nose.

Body Scan/Savasana - Sitting or mendacity down in a relaxed role (savasana is the "corpse pose"), one has to take a minute or two to breathe deeply and relax. Closing eyes and bringing your focal point to this present moment and your bodily self. A rapid body scan can take 30 seconds. A longer physique scan can take 30 minutes. Based on wants and circumstances, this exercising can be very flexible.

Visualization - Sitting in a at ease position, start by taking a few deep breaths to calm and carry thought to the current moment. Begin to visualize a place or space where one can be at peace. One might also find it most helpful to imagine a new place, where one has in no way been. It can resemble something familiar, but be a unique space.

\section{Concentration/Dharana Meditation -} This shape of meditation focuses the attention on an "object." The object ought to be a true physical object, a sound, an image, a mantra, or simply about something else. The object of center of attention is now not the most essential piece. Instead, what is most vital is really the manner of gaining knowledge of to concentrate. Learning this talent will have its very own benefits: rest and stress reduction.

\section{Mindful Breathing/ Anapanasati -} commence by taking a few deep breaths to relax. - begin with respiratory commonly via nose and paying attention to your inhalations and exhalations. - One has to try to find a spot the place breath is most noticeable. May additionally desire to attempt counting your breaths (1-10 and then start over) may also choose to identify inhalations by way of announcing to oneself, "In" and title your exhalations through saying, "Out." - If want to use counting or naming, use them as tools to assist keep a focal point on your breathing. Do now not let them become the object of focus.

\section{Mindfulness Meditation/ Vipassana -} object of meditation is one's own consciousness. - use the breath as an anchor to the current moment, it is no longer the only object (as in other forms of meditation) - emerge as aware of whatever the most distinguished stimulus is in that moment, and we enable that stimulus to be our object- may also be a thought, a feeling, a physical sensation, a sound, a smell, or simply about whatever else. - Virtually be aware it barring becoming worried in questioning about it. In this sense, we are working towards accomplishing a goal and non-reactive state of mind. At the same time, Vipassana is no longer primarily a relaxation method and we are not making an attempt to flee from fact or go off into a trance. The intention is energetic and 
objective statement of our subjective experience, barring attachment.

\section{EMPHERICAL REVIEWS ON EFFECTIVENESS OF MCBT ON MENTAL AND PHYSICAL CONDITIONS}

\section{Mindfulness In Depression: A} current study examined an effect of MCBT and concluded that it is a wonderful intervention that promotes accelerated stages of self-compassion and emotional regulation. Engaging with Serene can also help limit depressive signs and symptoms through mindfulness, self-compassion, and cognitive restructuring which help limit over identification with one's negative emotions. As individuals rebalance their questioning via cognitive restructuring, they can pick out the various stressors in their life, increase action plans and engage in adaptive coping techniques to address them. Serene can also promote increased selfunderstanding which can also supply one with a greater balanced standpoint on their modern-day upsetting situations to positively transform their challenges all through the pandemic. ${ }^{11}$

Mindfulness In Anxiety disorder: Mindfulness-based interventions (MBIs) have emerged as clinically advantageous interventions for nervousness and depression though there are full-size boundaries to their access in the generic population. The current learns about examined the effectiveness of a 5-week abbreviated mindfulness-based cognitive remedy (MBCT) intervention for a physician-referred, treatment-seeking, community pattern $(\mathrm{N}=54)$ with mood and/or anxiousness symptom burden. Treatment effects tested significant reductions in mood and anxiety symptom severity and good sized increases in typical well-being. Observed effect sizes had been typically large, with high response and remission rates. The present learn about presents preliminary support that an abbreviated MBCT protocol can offer large cure results for reducing temper and anxiousness signs and ought to potentially offer an fantastic population-level strategy to enhance cost-effectiveness and get admission to care. ${ }^{12}$

\section{Mindfulness in Borderline Personality} disorder: A route analytic method was once used to have a look at the relationships of trait mindfulness with BPD features, interpersonal effectiveness, impulsive and passive emotion-regulation, and neuroticism in a psychiatric sample of adults $(\mathrm{N}=70)$. As hypothesized, mindfulness was once related inversely with BPD aspects and core areas of dysfunction, and these associations endured when controlling for neuroticism. Furthermore, mindfulness deficits continued to predict BPD elements even when interpersonal effectiveness, passive and impulsive emotion-regulation, and neuroticism had been controlled. These findings recommend that mindfulness may additionally be a special predictor for the expression of BPD pathology. An emphasis on mindfulness might also as a consequence be critical in bettering the method and therapy of BPD. ${ }^{13}$

Mindfulness in Anorexia Nervosa: An built-in review used to be performed by way of searching health care computerized databases to observe and summarize research of mindfulness in folks with anorexia nervosa and become aware of areas for future research. Results had been combined among the eight research that met inclusion criteria. Multimodal mindfulnessbased therapies appear effective, whilst brief interventions may additionally be equally beneficial or result in larger anxiety compared to distraction. Qualitative information aid that some participants sense mindfulness is challenging however beneficial. Mindfulness as a concurrent section of remedy and/or when routinely practiced may be extra clinically beneficial than single-episode conscious eating interventions. Due to the complexity of the 
notion of mindfulness and constrained existing data, extra lookup is needed. ${ }^{14}$

\section{Mindfulness in psycho-physiological}

disorder: Recovery from the syndrome requires interrupting the ache cycle. This entails fundamental elements, all of which can be supported by way of mindful practice: cognitive restructuring, resuming full physical endeavor, working with terrible emotions. Both mindfulness exercise and its related perception would be effective in resolving the muscle tension disorders, Gastrointestinal and dermatological disorders, sexual dysfunctions and insomnia.

\section{Mindfulness in Epilepsy Patients}

According to the highlights and report from NDTV, having an epileptic episode can be frightening and confusing for each the affected person and the caregiver. Hence, it is vital spread as a lot recognition and understanding about the condition, so that the caregivers can manage a sudden seizure attack anytime, anywhere. As a caregiver, it is needed to empathize and encourage a man or woman with epilepsy as well as let them recognize that their existence can be as profitable as everyone else's, which emphasized on practicing mindfulness everyday can substantially limit nervousness for both the affected person and the caregiver and ends in creating a wholesome mind. ${ }^{15}$

Mindfulness In Psychosis: A proof mapping approach used to systematically search and perceive where and how mindfulness research has been conducted throughout ranges of psychosis to examine for intervention techniques and protection concerns, and content analysis examined practitioner hints to discover guidelines inside and across levels of illness. A paucity of lookup referring to the application of mindfulness in the therapy of psychotic illness exists for the high danger stage, with some research in FEP. The majority of lookup examines mindfulness techniques for the chronic stage of illness. Intervention formats for unique ranges of psychosis varied widely. Across all stages of psychosis, authors endorsed adaptations for cognitive impairments (e.g., shorter exercises for impacted attention), longer interventions, and greater beneficial methods of explaining mindfulness and encouraging practice. Few stage-specific pointers emerged beyond the doable helpfulness of compassion-based practices within earlier stages of illness the place selfstigmatization often emerges. Recommendations for how to first-rate adapt mindfulness to early intervention in psychosis are needed, and future lookup wants to higher recognize dangers and utility of exclusive mindfulness practices for different degrees of illness. ${ }^{16}$

Mindfulness In Cancer: An systematic review was conducted on MindfulnessBased Interventions in Cancer Survivors through the usage of Four databases (PubMed, Psychology and Behavioral Sciences, PsycINFO, and CINAHL) posted earlier than October 15, 2020. Articles had been included if they evaluated the benefits of an MBI application for adults with cancer. Twenty-one studies $(\mathrm{N}=1811$ participants) assembly the inclusion criteria have been recognized (randomized managed trials $(n=13)$, non-randomized controlled designs $(n=2)$, single-group research $(n=6))$. The pooled adherence price for participants' domestic exercise was once $60 \%$ of the assigned amount, which equated to $27 \mathrm{~min}$ per day all through the intervention period. There was once some proof for a relationship between home exercise of mindfulness techniques and enhancements in mood, stress, anxiety, depression, and concern of cancer recurrence (correlation coefficients ranged from third to 0.67). The study concluded that adherence to mindfulness domestic exercise amongst cancer survivors is sub optimal, and most of the correlates of adherence studied to date are non-modifiable. More research is warranted to scrutinize the function of 
domestic exercise in mindfulness-based interventions, consisting of evaluation of modifiable elements influencing adherence to enhance benefits for this population. ${ }^{17}$

Mindfulness And Substance Use; A randomized control study was conducted on mindfulness-based intervention and its effects on substance use and relapse among women in residential treatment. The study intervention sessions attended (dose) correlated with a greater length of time to alcohol intoxication $(r=.48, p<.001)$, fewer days of alcohol intoxication $(r=$ $-.24, p=.020$ ), and greater improvement in mindfulness skills $(r=.61, p<.01)$. And concluded that mindfulness based intervention added to an ongoing intensive residential treatment program serving vulnerable women is protective against marijuana use but no other substance use outcomes. Mindfulness practice time predicted a delay in time to first marijuana use. MMWR class attendance, an indicator of intervention dose, appears protective of alcohol intoxication at follow-up; thus, extended MMWR exposure might be useful. ${ }^{18}$

Mindfulness And Sexual Disorder; A literature evaluate identified seven trials for sexual dysfunction and Mindfulness based interventions (MBI) among these, 4 trials have been Randomized Control Trials (RCTs) along with a control-group wait listing and three research used withinsubjects design. A meta-analysis synthesized the consequences across these trials which verified a basic low to reasonable effect dimension (Cohen's $\mathrm{d}=0.55$ ). Moderate heterogeneity between studies was evident with possible guide bias. Sub-group analysis revealed differences between study weights, the place universal greater effect sizes have been discovered amongst RCTs $(d=0.65)$ than inside problem diagram studies $(\mathrm{d}=0.27)$. However, within concern format studies tended to encompass pre and postphysiological measurements (e.g. vaginal photoplethysmography), which may also grant a more unique assessment of the benefits of MBI, alternatively than studies primarily based on self-report measures only. This evaluate identified a gender inequality in which medical trials predominated amongst ladies compared to men with a 6:1 ratio. Whilst MBI appears promising, extensively greater research is needed, particularly amongst men, so that science, exercise and coverage can develop an understanding of the attainable benefits of non-prescription intervention alternatives, or as a biopsychosocial adjunct, such as $\mathrm{MBI}$, in aid of those experiencing sexual dysfunction. ${ }^{19}$

Mindfulness in Premenstrual syndrome; Premenstrual syndrome (PMS) is a very common circumstance leading to physical, emotional, and psychological misery in ladies in the course of their reproductive years. Researchers observed that as many three of every 4 menstruating girls have experienced some shape of PMS. Although the severity of this trouble is great, a lot of confusion exists in both scientific and lay communities about what is and is not effective for the therapy of PMS. It has a huge range of symptoms and signs and symptoms and it can also tend to recur in a predictable pattern. The bodily and emotional changes experienced with PMS may fluctuate from just barely great to very intense. Lifestyle adjustments in each and every sort can help to reduce or manipulate the signs and symptoms and of PMS. Apart from all these, one of the most recommended techniques to limit the difficulties of PMS is rest therapy which consists of meditation, visualization, autogenic, Tai Chi, hydrotherapy, exercise, massage, aromatherapy, biofeedback, relaxation breathing, and revolutionary muscle leisure (Jacobson's modern rest therapy and The Mitchells modern leisure therapy) and yoga. ${ }^{20}$ 


\section{MINDFULNESS IN DAILY LIFE}

Mindfulness is the energy of being aware and awake to the present moment. It is the continuous practice of touching life deeply in every moment of daily life. To be mindful is to be truly alive, present, and at one with those around you and with what you are doing. We bring our body and mind into harmony while we do are daily routines. ${ }^{21}$ As well as setting aside time for a more formal practice, you can practice mindfulness in daily life. For example: Notice everyday things like the air moving past you as you move, the feel of a banister as you go upstairs, or the taste and textures of the food you eat. Pick a regular time such as your morning commute or evening walk - to be more aware of the sensations caused by the world around you. Try something new, like a different route home from the station or a different seat on the bus. This can help you notice the world in a different way. Watch your thoughts. If you sit quietly for a few moments, you will probably notice thoughts drifting through your mind. Instead of engaging with them, simply observe them and let them go. Research has highlighted that in order to sustain positive brain changes, we need: sustained commitment, mentor and peer support, and experiential integration.
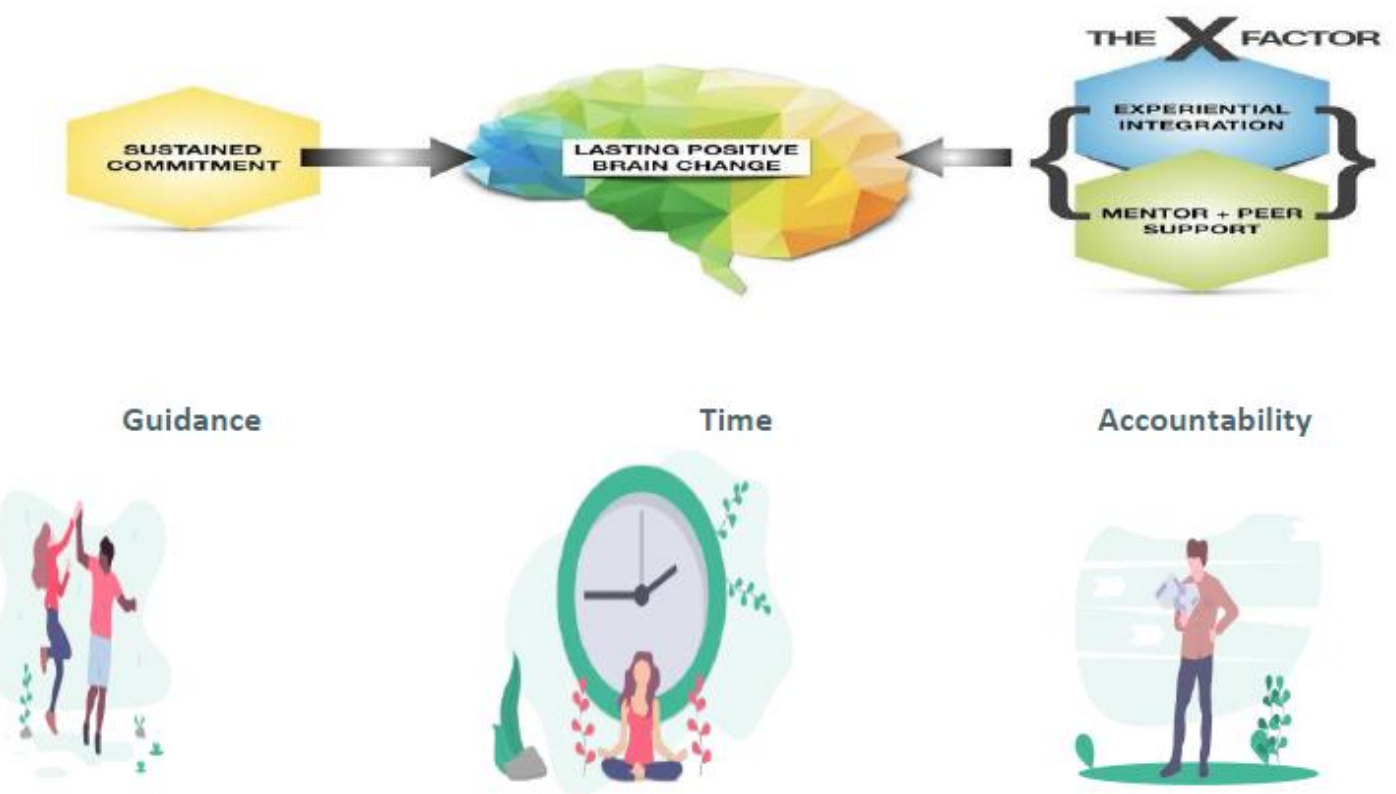

Accountability

Guidance: Support and accountability are essential to helping you maintain your momentum.

Time: To establish the new habit, and really integrate your new learning into your day to day routines.

Accountability: From those who have been there before, and can show you proven methods that work

\section{LIMITATIONS AND CONCERNS}

The effectiveness of mindfulness based cognitive remedy is supported with the aid of using enormous empirical proof and has. in step with studies, normally produced wonderful effects for humans in remedy. However, due to the fact it's miles a distinctly new remedy modality, the longtime period blessings of this method might not but be absolutely determinable. A developing frame of empirical proof helps the method, however similarly studies may also offer more assist for its effectiveness while treating bipolar, consuming issues, psychosis, and different conditions. Several critics have highlighted methodological shortcomings in a few MBCT research which includes small pattern size, a loss of manage groups, and a loss of randomization, all of that have capacity to have an effect on the effects obtained. More rigorous research has to compare whether or not MBCT is 
extra powerful than different, extra broadly practiced kinds of remedy, which includes cognitive behavioral remedy.

\section{CONCLUSION}

Mindfulness education gives one tool of many to facilitate both the stabilization and destabilization components of change. Enthusiasm for mindfulnessbased interventions is balanced with the aid of enormous skepticism. Empirical evidence such as systematic reviews of RCTs has indicated that MBCT is an effective intervention on this context. Despite robust help in its want, this frame of studies is not without its limitations. Much remains to be worked out at theoretical and realistic degrees. Future of mindfulness-based remedies is likely to rely on demonstrations of their distinct, nice and lasting contributions that other clinicians cannot bypass. In order to utilize Mindfulness into practice, therapists must have an honest understanding of the disorders they're treating, also as knowing how Mindfulness are often helpful with those disorders. In using Mindfulness techniques, therapists must have an honest understanding of Mindfulness, while also having received formal training. Therapists should practice mindfulness themselves as a way of appropriate modeling to their clients. Possible unintended negative effects may include increased restlessness, anxiety, depression, guilt, and hallucinations. Mindfulness training may only be helpful in certain situations. Thus, therapists got to be conscious of the restrictions of Mindfulness and when it is/is not appropriate to be used. As an advancing approach, mindfulness has advertised a heap of scales to degree the therapeutic results. Utilizing self-report has been thought as the suitable strategy because it is impossible to degree mindfulness objectively Professionals can select one of the available scales concurring to their treatments, clients, and the issues that their clients endure from. In expansion, given the truth that there are comparable concepts of mindfulness in other religious traditions, future investigate may dive into this issue to enhance the current mindfulness perspective. It is additionally critical to conduct considers to compare the viability of certain treatments with a group of members from diverse societies and religions. Moreover, future inquire about might focus on the applications of mindfulness values to the world, such as sympathy, interest, non judgmental, and loving kindness. It appears that these mindfulness values are as of now required by the world amid this time

\section{Acknowledgement: None}

Conflict of Interest: None

Source of Funding: None

Ethical Approval: Not Applicable

\section{REFERENCES}

1. Sipe, W.E., \& Eisendrath, S.J. (2012). Mindfulness-based cognitive therapy: Theory and practice [Abstract]. Canadian Journal of Psychiatry, 57(2), 63-69. Retrieved from http://www.ncbi.nlm.nih.gov/pubmed/ 22340145

2. Quotes from Gethin, Rupert M.L. (1992), The Buddhist Path to Awakening: A Study of the BodhiPakkhiȳa Dhammā. BRILL's Indological Library, 7. Leiden and New York: BRILL

3. Brown, K. W., \& Cordon, S.Toward a phenomenology of mindfulness: Subjective experience and emotional correlates. In F. Didonna (Ed.), Clinical handbook of mindfulness (pp. 59-81) 2009, New York, NY: Springer-Verlag.

4. Kabat-Zinn- Mindfulness Training as a Clinical Intervention: A Conceptual and Empirical Review, by Ruth A. Baer, available at http://www.wisebrain.org /papers/MindfulnessPsyTx.pdf. 1994 
5. Hyland, Terry, On the Contemporary Applications of Mindfulness: Some Implications

6. For Education, Journal of Philosophy of Education, Vol. 49, No. 2, 2015 On the Contemporary Applications of Mindfulness: Some Implications for Education DO - 10.1007/978-94-0071911-8_3

7. Mark Williams J G, Jon Kabat-Zinn , Mindfulness Diverse Perspectives on its Meaning, Origins and Applications, ISBN 9780415636476 Published February 12, 2013 by Routledge,1st Ed ,328 Pages, Copyright Year 2013.

8. Teasdale, J.D., Williams, J.M.G., Segal, Z.V. (2014). The Mindful Way Workbook: An 8-Week Program to Free Yourself from Depression and Emotional Distress. Guilford Press. https://en.wikipedia.org/wiki/Mindfulne Ss

9. Shian-LingKeng Moria J.Smoski Clive J.Robins , Effects of mindfulness on psychological health: A review of empirical studies Clinical Psychology Review Volume 31, Issue 6, August 2011, Pages 1041-1056. https://doi.org/10.1016/j.cpr.2011.04.00 6

10. Carmody, James, Baer, Ruth, Relationships between mindfulness practice and levels of mindfulness, medical and psychological symptoms and well-being in a mindfulness-based stress reduction program, Journal of behavioral medicine, March 2008 ,31(1):23-33DO - 10.1007/s10865-0079130-7

11. WillemKuyken ,EdWatkins etal ,How does mindfulness-based cognitive therapy work?Behaviour Research and Therapy,Volume 48, Issue 11, November 2010, Pages 1105-1112 https://doi.org/10.1016/j.brat.2010.08.00 3

12. Crane,R. (2009). Mindfulness-based cognitive therapy: Distinctive features. Routledge/Taylor \& Francis
Group. American

Psychological Association. ISBN: 0415445027

13. MohamedAl-Refae, etal A SelfCompassion and Mindfulness-Based Cognitive Mobile Intervention (Serene) for Depression, Anxiety, and Stress: Promoting Adaptive Emotional Regulation and Wisdom .Front. Psychol., 22

March 2021https://doi.org/10.3389/fpsy g.2021.648087

14. Burgess, E.E., Selchen, S., Diplock, B.D. et al. A Brief Mindfulness-Based Cognitive Therapy (MBCT) Intervention as a Population-Level Strategy for Anxiety and Depression. J Cogn Ther 14, 380-398 (2021). https://doi.org/10.1007/s41811-02100105

15. Wupperman P, Neumann CS, Whitman JB, Axelrod SR. The role of mindfulness in borderline personality disorder features. J Nerv Ment Dis. 2009 Oct;197(10):766-71. doi: 10.1097/NMD.0b013e3181b97343. PMID: 19829206.

16. Julie Dunne, Mindfulness in Anorexia Nervosa: An Integrated Review of the Literature,J Am Psychiatr Nurses Assoc ,Mar/Apr 2018;24(2):109-117.doi: $10.1177 / 1078390317711250$.

17. Sneha George, International Epilepsy Day 2021: 8 Things Caregivers of Epileptic Patients Need To Follow, As Per Expert Updated: February 08, 2021 6:10 pm IST

18. Reich, D., Evans, S., Nelson, B. et al. Evidence Map of Mindfulness for Stages of Psychosis: State of the Literature and Implications for Future Research. Mindfulness 12, 1860-1877 (2021). https://doi.org/10.1007/s12671021-01611.

19. Baydoun M, Moran C, McLennan A, Piedalue KAL, Oberoi D, Carlson LE. Mindfulness-Based Interventions in Cancer Survivors: A Systematic Review of Participants' Adherence toHome Practice. Patient Prefer Adherence. 
Bemina JA et.al. Glimpse of contemporary clinical perspective on "mindfulness based cognitive behavioral therapy" in various health dimensions.

2021;

$15: 1225-1242$. https://doi.org/10.2147/PPA.S267064

20. Amaro, Hortensia PhD; Black, David S. PhD Mindfulness-Based Intervention Effects on Substance Use and Relapse Among Women in Residential Treatment: A Randomized Controlled Trial With 8.5-Month Follow-Up Period From the Moment-by-Moment in Women's Recovery Project, Psychosomatic Medicine: 7/8 2021 Volume 83 - Issue 6 - p 528-538. doi: 10.1097/PSY.

21. Banbury, S., Lusher, J., Snuggs, S., \& Chandler, C. Mindfulness-based therapies for men and women with sexual dysfunction: A systematic review and meta-analysis. Sexual and
Relationship Therapy, Advance online publication, (2021). https://doi.org/10.1080/14681994.2021

22. Ancy Jose, etal. The Mystery behind Relaxation Therapy: Adieu to Premenstrual Syndrome, Journal of Health and Allied Sciences, Vol. 00 No. 0/2021,2021.DOI https://doi.org/ 10.1055/s-0041-1726689

How to cite this article: Bemina JA, Theraza Mathais. Glimpse of contemporary clinical perspective on "mindfulness based cognitive behavioral therapy" in various health dimensions. International Journal of Research and Review. 2022; 9(2): 236-246. DOI: https://doi.org/10.52403/ijrr.20220233 